\title{
Electromagnetic Characteristics Measurement Setup at Variable Temperatures Using a Coaxial Cell
}

\author{
Thibaut Letertre $\mathbb{D}^{\mathrm{D}},{ }^{1}$ Philippe Pouliguen (D), $^{2}$ and Pierre Sabouroux (D) $^{1}$ \\ ${ }^{1}$ Aix Marseille Univ, CNRS, Centrale Marseille, Institut Fresnel, Marseille, France \\ ${ }^{2} D G A$, Balard, Paris, France \\ Correspondence should be addressed to Thibaut Letertre; thibaut.letertre@fresnel.fr
}

Received 17 January 2019; Revised 25 March 2019; Accepted 8 April 2019; Published 8 May 2019

Academic Editor: Matjaz Valant

Copyright (C) 2019 Thibaut Letertre et al. This is an open access article distributed under the Creative Commons Attribution License, which permits unrestricted use, distribution, and reproduction in any medium, provided the original work is properly cited.

\begin{abstract}
This article presents an easy way to measure frequency dependence of the complex permittivity and complex permeability for any kind of material from $10 \mathrm{MHz}$ to $6 \mathrm{GHz}$, with temperature variation between the ambient temperature and $85^{\circ} \mathrm{C}$. This work is based on a well-known transmission/reflection technique using a coaxial line equipped with a thermoregulation system to manage temperature variations in the sample confinement area. The paper underlines some effects which have to be taken into account with temperature variation. APU10 and cyclohexanol are presented as examples of solid and liquid reference materials.
\end{abstract}

\section{Introduction}

Measuring the electromagnetic properties of materials is widely studied nowadays. In the last twenty years, many useful measurement methods in microwave range [1] have been developed for specific applications. Waveguide [2], as the transmission/reflection method, resonators [3, 4], or free space [5] are the most known techniques. These devices are designed to operate at room temperature over a specific frequency range. In many cases, it becomes important to determine the dependence of dielectric quantities as a function of temperature, up to temperatures that can be very high (several hundred degrees in the case of aircraft materials located near aircraft engines). In this context, a new setup, using a coaxial line [6] equipped with a wideband thermoregulated PID system to manage temperature variations in the sample area is proposed. In the literature, a few setups have been proposed to study the temperature behaviour of materials, but mainly using the resonator $[4,7,8]$ or free space methods to reach high temperature [9]. Nonetheless, in these cases, it is not possible to measure on a wideband of frequency. A few studies of waveguided setups in temperature have been done [10], but these techniques are often complex and bulky. That is why we decided to propose an easy-to-use solution to measure the permittivity and permeability of any kind of material based on a coaxial line [2], with temperature variation between ambient and $85^{\circ} \mathrm{C}$. This paper is divided into two parts; the first part is focused on the frequency measurement method, followed by the temperature variation method. The second part presents the results obtained on a few different materials. The first reference material is a solid magnetic sample, a silicon-metal composite. As a second reference material, cyclohexanol, which is a dielectric alcohol well known in the literature [11], is used.

\section{Measurement Method}

In this section, the different processes to determine the frequency and temperature dependence of the electromagnetic characteristics of materials are detailed. We start with a brief definition of the classical mathematical equations and of an uncertainty estimation for the frequency dependence of the complex permittivity and the complex permeability. Afterwards, we propose a process to accurately manage the temperature in the sample to obtain the permittivity and permeability behaviours. 


\subsection{Frequency Measurement}

2.1.1. Mathematical Equations. The permittivity and permeability are defined as complex values:

$$
\begin{aligned}
& \varepsilon=\left[\varepsilon_{\mathrm{R}}^{\prime}-j \varepsilon_{\mathrm{R}}^{\prime \prime}\right] \varepsilon_{0}=\varepsilon_{\mathrm{R}}^{*} \varepsilon_{0}, \\
& \mu=\left[\mu_{\mathrm{R}}^{\prime}-j \mu_{\mathrm{R}}^{\prime \prime}\right] \mu_{0}=\mu_{\mathrm{R}}^{*} \mu_{0},
\end{aligned}
$$

where $\varepsilon_{\mathrm{R}}^{\prime}$ and $\mu_{\mathrm{R}}^{\prime}$ correspond to the relative permittivity and relative permeability. On the other hand, $\varepsilon_{0}$ and $\mu_{0}$ correspond to the vacuum permittivity and vacuum permeability, respectively.

The setup is composed by a vector network analyser (Anritsu MS2038 C) and a tapered coaxial line. The coaxial line is divided into three parts.

The two tapered parts were designed to convert the $7 \mathrm{~mm}$ diameter line of the coaxial connector, into a $13 \mathrm{~mm}$ line, to significantly increase the sample volume. Furthermore, assuming the conical part as $50 \Omega$ lines, it is possible to use a de-embedding method [2] to obtain the $S$-parameters of the sample.

The relation between the $S$-parameters and the transmission parameter $z$ and the reflection parameter $\Gamma$ of a sample are

$$
\begin{aligned}
& S_{21}=\frac{\left(1-\Gamma^{2}\right) z}{1-(\Gamma z)^{2}}, \\
& S_{11}=\frac{\left(1-z^{2}\right) \Gamma}{1-(\Gamma z)^{2}},
\end{aligned}
$$

where $z$ and $\Gamma$ can be expressed as

$$
\begin{aligned}
z^{2} & =\frac{S_{11}-\Gamma}{\Gamma^{2} S_{11}+\Gamma} \\
\Gamma & =\frac{1-S_{21}^{2}+S_{11}^{2}}{2 S_{11}} \pm \sqrt{\left(\frac{1-S_{21}^{2}+S_{11}^{2}}{2 S_{11}}\right)^{2}-1 .}
\end{aligned}
$$

With $|\Gamma| \leq 1$. Next, the refraction index $n$ and the line impedance $Z$ are calculated:

$$
\begin{aligned}
& Z=\frac{1+\Gamma}{1-\Gamma}=\sqrt{\frac{\mu}{\varepsilon}}, \\
& n=\frac{c_{0} k}{\omega}=\sqrt{\mu * \varepsilon} .
\end{aligned}
$$

$k$ can be expressed as

$$
k=-\frac{\ln z}{j d} .
$$

Finally, with equation (1), permittivity and permeability are obtained:

$$
\begin{aligned}
\varepsilon & =\frac{n}{Z}, \\
\mu & =n * Z .
\end{aligned}
$$

This method is useful to directly obtain the permittivity and permeability without any assumption on the material properties. Hence, the study of any kind of material (liquid, solid, powder, etc.) is possible.

2.1.2. Uncertainty Estimation. In transmission/reflection method, we expect different sources of errors:

(i) Errors in measuring $S$-parameter

(ii) Gaps between sample and sample holder

(iii) Uncertainties in the length of the sample

(iv) Uncertainties in the plane reference position

In our case, $S$-parameter measurement uncertainties have been obtained in the datasheet manual of the vector network analyser (Anritsu MS2038C). Those errors only depend on the calibration. Samples have been made carefully in order to fit perfectly to the sample holder. In this way, we do not care about gaps in this uncertainties model. While the $S$-parameter errors can be compared as offset error in our measurement, the length's uncertainties and the reference position uncertainties are difficult to determine. The reference positions are presented in this paper with the de-embedding method. This method transforms the $S$-parameters of the cell into the $S$-parameters of the sample because the sample is locked up in the middle of the cell. We define 2 errors for 2 different lengths $\left(l_{1}\right.$ and $\left.l_{2}\right)$ of the conical parts (Figure 1) $\Delta l_{1}$ and $\Delta l_{2}$. Furthermore, to obtain the permittivity and the permeability of the sample, its length is needed. Because of this, we have to consider the error of the length of the sample $\Delta L$. In order to estimate the uncertainties of the $S$-parameter of the sample considering the error of the $S$-parameter, $\Delta l_{1}, \Delta l_{2}$, and $\Delta L$, we derive the equations of the de-embedding method [2]. We assume that the total error of the de-embedded $S$-parameter can be written as

$$
\begin{aligned}
\Delta S_{11}^{\mathrm{de}} & =\sqrt{\left(\frac{\partial S_{11}^{\mathrm{de}}}{\partial\left|S_{11}\right|} \Delta\left|S_{11}\right|\right)^{2}+\left(\frac{\partial S_{11}^{\mathrm{de}}}{\partial \theta_{11}} \Delta \theta_{11}\right)^{2}+\left(\frac{\partial S_{11}^{\mathrm{de}}}{\partial l_{1}} \Delta l_{1}\right)^{2}} \\
\Delta S_{21}^{\mathrm{de}} & =\sqrt{\left(\frac{\partial S_{21}^{\mathrm{de}}}{\partial\left|S_{21}\right|} \Delta\left|S_{21}\right|\right)^{2}+\left(\frac{\partial S_{21}^{\mathrm{de}}}{\partial \theta_{21}} \Delta \theta_{21}\right)^{2}+\left(\frac{\partial S_{21}^{\mathrm{de}}}{\partial l_{1}} \Delta l_{1}\right)^{2}+\left(\frac{\partial S_{21}^{\mathrm{de}}}{\partial l_{2}} \Delta l_{2}\right)^{2}}
\end{aligned}
$$




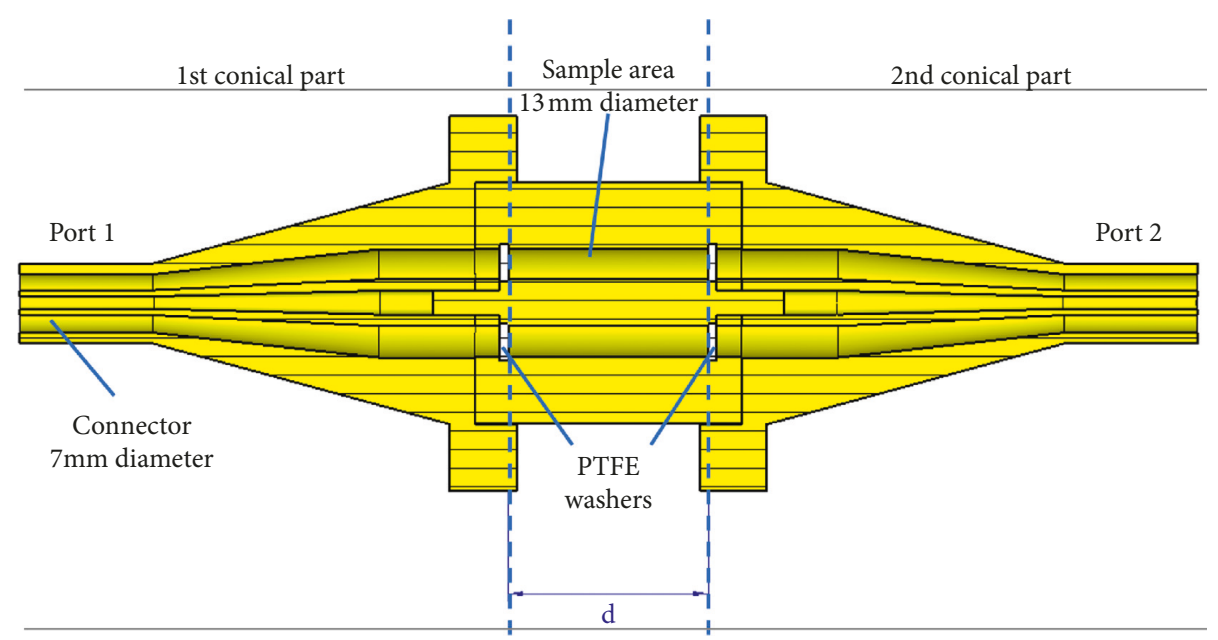

Figure 1: Measurement coaxial line. CST design.

where $\Delta\left|S_{11}\right|, \Delta\left|S_{21}\right|, \Delta\left|\theta_{11}\right|$, and $\Delta\left|\theta_{21}\right|$ correspond to the uncertainties of the magnitude and the phase of the $S$ parameter of the cell. $\Delta\left|S_{11}^{\mathrm{de}}\right|, \Delta\left|S_{21}^{\mathrm{de}}\right|, \Delta\left|\theta_{11}^{\mathrm{de}}\right|$, and $\Delta\left|\theta_{21}^{\mathrm{de}}\right|$ correspond to the uncertainties of the magnitude and the phase of the $S$-parameter of the sample. Finally, we estimate the error of the permittivity and the permeability as a function of the error of the de-embedded $S$-parameter and the error of the sample's length [12]:

$$
\begin{aligned}
& \frac{\Delta \varepsilon_{\mathrm{R}}^{\prime}}{\varepsilon_{\mathrm{R}}^{\prime}}=\frac{1}{\varepsilon_{\mathrm{R}}^{\prime}} \sqrt{\left(\frac{\partial \varepsilon_{\mathrm{R}}^{\prime}}{\partial\left|S_{\alpha}^{\mathrm{de}}\right|} \Delta\left|S_{\alpha}^{\mathrm{de}}\right|\right)^{2}+\left(\frac{\partial \varepsilon_{\mathrm{R}}^{\prime}}{\partial \theta_{\alpha}^{\mathrm{de}}} \Delta \theta_{\alpha}^{\mathrm{de}}\right)^{2}+\left(\frac{\partial \varepsilon_{\mathrm{R}}^{\prime}}{\partial L} \Delta L\right)^{2}}, \\
& \frac{\Delta \varepsilon_{\mathrm{R}}^{\prime \prime}}{\varepsilon_{\mathrm{R}}^{\prime \prime}}=\frac{1}{\varepsilon_{\mathrm{R}}^{\prime \prime}} \sqrt{\left(\frac{\partial \varepsilon_{\mathrm{R}}^{\prime \prime}}{\partial\left|S_{\alpha}^{\mathrm{de}}\right|} \Delta\left|S_{\alpha}^{\mathrm{de}}\right|\right)^{2}+\left(\frac{\partial \varepsilon_{\mathrm{R}}^{\prime \prime}}{\partial \theta_{\alpha}^{\mathrm{de}}} \Delta \theta_{\alpha}^{\mathrm{de}}\right)^{2}+\left(\frac{\partial \varepsilon_{\mathrm{R}}^{\prime \prime}}{\partial L} \Delta L\right)^{2}}, \\
& \frac{\Delta \mu_{r}^{\prime}}{\mu_{r}^{\prime}}=\frac{1}{\mu_{r}^{\prime}} \sqrt{\left(\frac{\partial \mu_{r}^{\prime}}{\partial\left|S_{\alpha}^{\mathrm{de}}\right|} \Delta\left|S_{\alpha}^{\mathrm{de}}\right|\right)^{2}+\left(\frac{\partial \mu_{r}^{\prime}}{\partial \theta_{\alpha}^{\mathrm{de}}} \Delta \theta_{\alpha}^{\mathrm{de}}\right)^{2}+\left(\frac{\partial \mu_{r}^{\prime}}{\partial L} \Delta L\right)^{2}}, \\
& \frac{\Delta \mu_{\mathrm{R}}^{\prime \prime}}{\mu_{\mathrm{R}}^{\prime \prime}}=\frac{1}{\mu_{\mathrm{R}}^{\prime \prime}} \sqrt{\left(\frac{\partial \mu_{\mathrm{R}}^{\prime \prime}}{\partial\left|S_{\alpha}^{\mathrm{de}}\right|} \Delta\left|S_{\alpha}^{\mathrm{de} e}\right|\right)^{2}+\left(\frac{\partial \mu_{\mathrm{R}}^{\prime \prime}}{\partial \theta_{\alpha}^{\mathrm{de}}} \Delta \theta_{\alpha}^{\mathrm{de}}\right)^{2}+\left(\frac{\partial \mu_{\mathrm{R}}^{\prime \prime}}{\partial L} \Delta L\right)^{2}},
\end{aligned}
$$

where $\alpha=11$ or 21 corresponding to the reflection and the transmission parameter. Among all these error sources, we studied especially the error of the sample's length which is measured manually with a caliper. Considering the mechanical nature (rigidity) of the material to characterize, the accuracy of the thickness determination using a caliper can then be obtained between 0.5 and $0.1 \mathrm{~mm}$. We compared the two of them to show the impact on the general error (Figure 2).

We easily understand how it became important to have a good precision of the sample length. We did not plot the impact of the error of 11 and 12 , but the higher the precision,

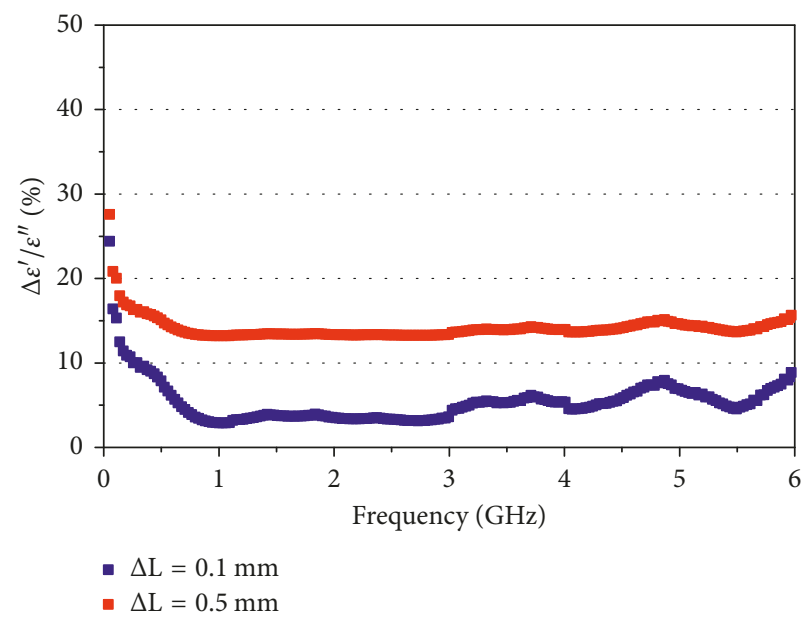

FIGURE 2: Relative uncertainty in $\varepsilon_{\mathrm{R}}^{\prime}$ as a function of frequency where $\varepsilon_{\mathrm{R}}^{\prime}$ is the result of a permittivity measurement of a $4 \mathrm{~mm}$ length sample of APU10 (solid magnetic material presented in Results).

the lower the error. Furthermore, Using a transmission reflection method, we can expect an error around 1\% [1] for the real part of the permittivity. In our case, we have approximately $5 \%$, which is acceptable. Considering that materials are impacted by temperature variation, the setup is updated with a temperature thermoregulation system to control the sample's temperature value.

2.2. Temperature Regulation System. A heating ring is used to manage the temperature variation of the coaxial line, which is placed around the sample holder. This ring is connected to a PID thermoregulation system.

With the present setup (Figure 3), measuring the permittivity and permeability of materials at any controlled temperature is straightforward. Nevertheless, the distribution of temperature homogeneity throughout the material and the dilation of the cell need to be assessed. 


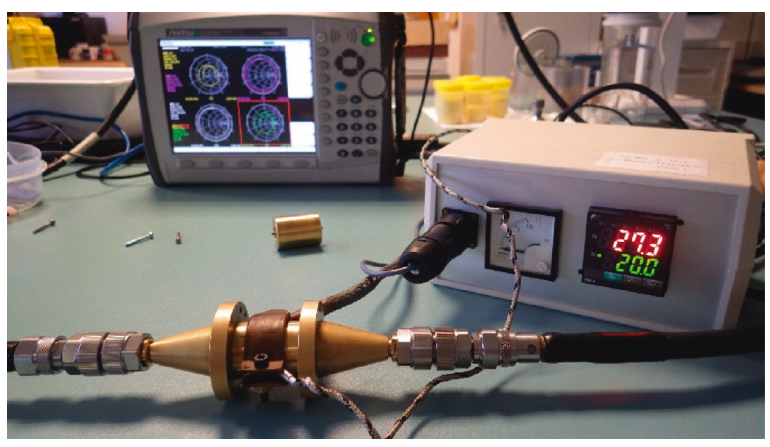

(a)

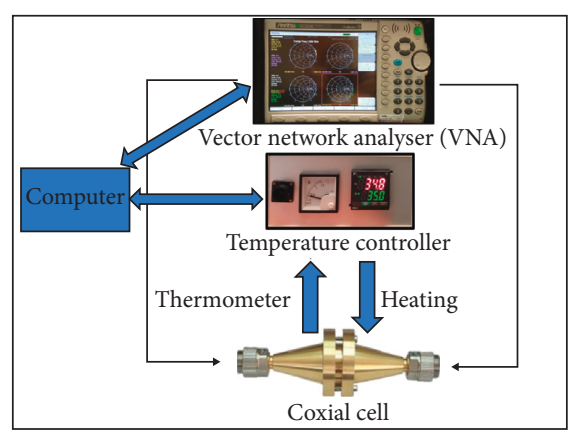

(b)

FIgURE 3: Picture and synoptic of the permittivity measurement setup with temperature variation.

2.2.1. Cell Dilation Effect. Heating of the sample holder increases the temperature of the entire coaxial cell. Thus, the cell can expand and directly modify the measured $S$-parameters. So, the permittivity and permeability, directly obtained by the $S$-parameters, also show the impact done by the temperature of the cell. To prove that the temperature has no dilation impact on the cell, an evaluation, as function of temperature, was carried out for the empty cell, i.e., the electromagnetic properties of air were measured with increasing temperature at a specific frequency. Figure 4 represents the real part of the permittivity of the air sample as a function of temperature at $1 \mathrm{GHz}$.

The literature shows that the permittivity of air is closed to 1 [13] and can be considered as a constant with temperature variation [14]. In the case where no dilation occurs in the cell, no variation of permittivity should be noticed and only the cell can change the permittivity measurement. We can notice that the variation of the real part of the permittivity is quite steady (Figure 4). The variation of the permittivity is about less than 1 percent of the permittivity of the air. This is insignificant compared to the error of the measurement technique. Therefore, it is possible to consider that the temperature has no impact on the coaxial line in this range of temperature.

2.2.2. Sample Homogeneity Effect. To accurately measure the correct temperature dependence of the permittivity and permeability, homogeneity of the temperature inside the material becomes important. To evaluate the temperature of the sample holder, a PT100 sensor is placed between the heating ring and the external part of the sample holder. We extrapolate the stabilization of the internal temperature of the sample holder, and thus of the sample itself, by applying a sufficient stabilization delay. This extrapolation generates a certain difference that disappears with sufficient waiting time (Figure 5(a)).

Considering only the temperature of the sample holder, it is impossible to precisely know the sample's temperature. The difference showed (Figure 5(a)) corresponds to the inhomogeneity of the temperature in the sample which is different from the temperature obtained by the PT100 sensor. Waiting for a time " $t$ ", depending on the material, until the sample is at the good temperature, is an easy way to correct

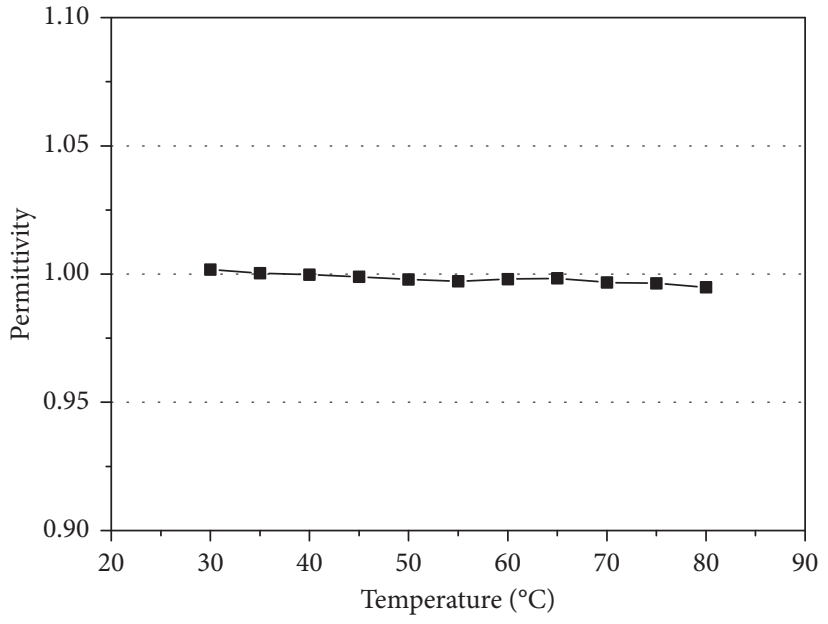

FIgURE 4: Picture of the real part of permittivity of air for an empty cell as a function of temperature at $1 \mathrm{GHz}$.

this inhomogeneity. This time corresponds to the moment when the temperature is the same, in the material and around the sample holder. It is called stabilization delay (SD) in this paper. Then, after the SD, the temperature inside the material can be considered homogeneous and the permittivity obtained could be assigned to the temperature measured.

The way to determine the SD depends on the material to test; the SD has to be defined correctly. In the Figure 5(b), the $\mathrm{SD}$ is evaluated with a measurement of permittivity between two steps of temperature as a function of time. The SD is fixed for a step of temperature (here, $5^{\circ} \mathrm{C}$ ) and is obtained when the permittivity does not change anymore. On liquids, permittivity evolves rapidly with temperature variation, especially in the case of alcohols. This process is not as straightforward when refering to solids or powders. To calculate the SD, when no variation of permittivity is noticed between two steps of temperature, a minimum time of $720 \mathrm{~s}$ is fixed for a step of $5^{\circ} \mathrm{C}$.

The thermoregulation system is designed to reach hundreds of degrees. However, in the actual configuration, the temperature is limited to $85^{\circ} \mathrm{C}$. This maximum is set by the maximum working temperature of the connectors placed between the VNA's N-type cables and the cell. To avoid irreversible problems on connectors and coaxial cables, the 


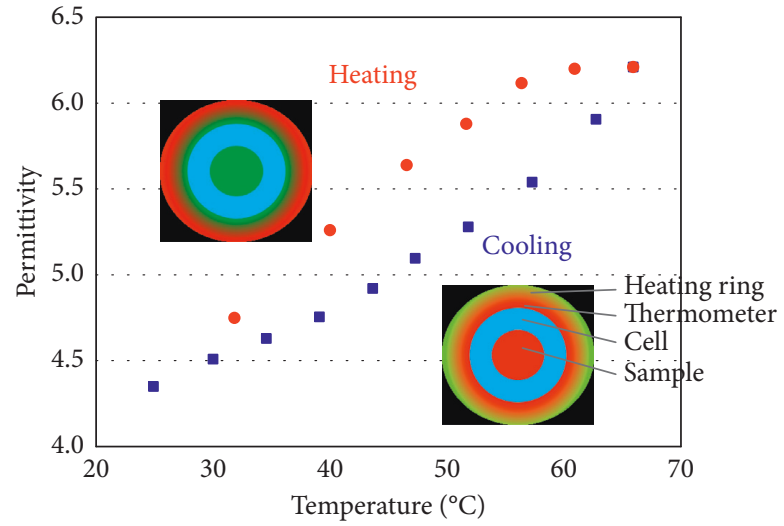

(a)

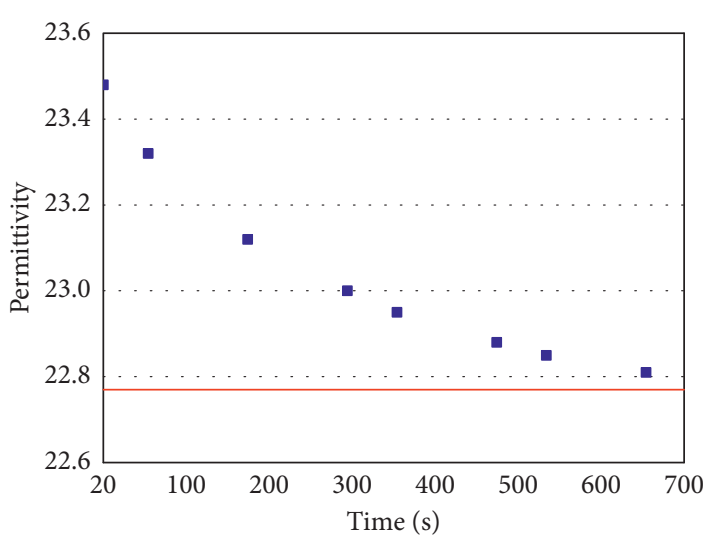

(b)

FIGURE 5: (a) Graphic representing the real part of permittivity of a sample of octanol with a positive and negative slope of temperature variation. (b) Graphic representing the real part of permittivity of ethanol at $100 \mathrm{MHz}$ as a function of time. It shows a heating-up process where at the beginning, the temperature was $30^{\circ} \mathrm{C}$ and after $600 \mathrm{~s}, 35^{\circ} \mathrm{C}$.

maximum temperature of the setup is $85^{\circ} \mathrm{C}$. The setup's performance has been checked from ambient to $85^{\circ} \mathrm{C}$.

\section{Results}

Thanks to the frequency measurement part and temperature measurement part, it becomes easy to characterize any kind of material on a wide range of frequency and between ambient temperature and $85^{\circ} \mathrm{C}$ with the coaxial line (Figure 1). Validation results are presented with two different reference materials on a wide band of frequency and at different temperatures. Due to the possibility to characterize any kind of materials, a solid magnetic material, APU10 (SIEPEL, France), and a nonmagnetic liquid, cyclohexanol, have been selected. Cyclohexanol is a well-known alcohol referenced in literature [11]. The principal aim of this work is to show that the setup is convenient for any application and can be easily configured in terms of frequency and temperature, bearing in mind that the applications are in the working range of this setup.

Figure 6 shows the permittivity and permeability of APU10. Figures 6(a) and 6(b) were generated with the same configuration and at the same time. The length of the sample was $4 \mathrm{~mm}$ (Figure 1). Two different temperatures have been chosen, $25^{\circ} \mathrm{C}$ as the ambient temperature, and $85^{\circ} \mathrm{C}$, which is the maximum temperature of the setup. The frequency range is $50 \mathrm{MHz}$ to $6 \mathrm{GHz}$. Thus, a full map of the frequency and temperature dependence of electromagnetic characteristics of the sample is obtained.

In Figure 6, the permittivity behaviour of the APU10 seems to be steady along the frequency, whereas the permeability decreases linearly. But, some wavy dependence appears on the measurement. We think that this phenomenon is produced by a slight mismatch in the coaxial line. As a carbonyl iron in a silicon matrix, the behaviour of the measurement can be compared to a classic carbonyl iron material. Considering our error, we assume that the permittivity is steady and the real part of the permittivity decreases upon 1 . The same measurement has been made with rectangular waveguide in the literature [15], which confirms the behaviour found.

In the same way, Figure 7 represents the permittivity and permeability of cyclohexanol. As in the APU10 case, the same frequency range and frequency step are used. The sample area was completely filled by cyclohexanol since the liquid is surrounded with the watertight Teflon walls of the sample holder. Differently from APU10, cyclohexanol expands with temperature dependence. Due to the Teflon washers (Figure 1) confining the liquid, pressure increases with temperature increase, until the washers give way. To avoid this phenomenon in the sample area, the maximum reached temperature was $45^{\circ} \mathrm{C}$ instead of $85^{\circ} \mathrm{C}$.

In Figure 7 , the permittivity of the cyclohexanol has a classic behaviour of many liquids as the alcohol. Indeed, we observe a relaxation dispersion which can be well fitted by a Cole-Cole model [16]. The real part of the permittivity seems to decrease fastest when we decrease the temperature to lower frequency. This effect is well known in the literature and is related to the relaxation time [17-19]. Decreasing the temperature freezes the electric dipoles in the alcohol and increases the relaxation time. It can be well explained by the "Arrhenius law" [20]. Furthermore, as a nonmagnetic material, we have a steady behaviour around 1 for the real part of permeability which confirms the validity of the measurement.

\section{Discussion}

This article presents a study on electromagnetic characteristic measurement with temperature variation. The goal of this study was to validate a wide-band measurement technique with temperature variation. The frequency measurement is obtained with a classical coaxial transmission line method, and the temperature measurement is managed with a thermoregulation system. The maximum temperature was set to the maximum temperature accepted by the connectors even if the actual setup could go further. 


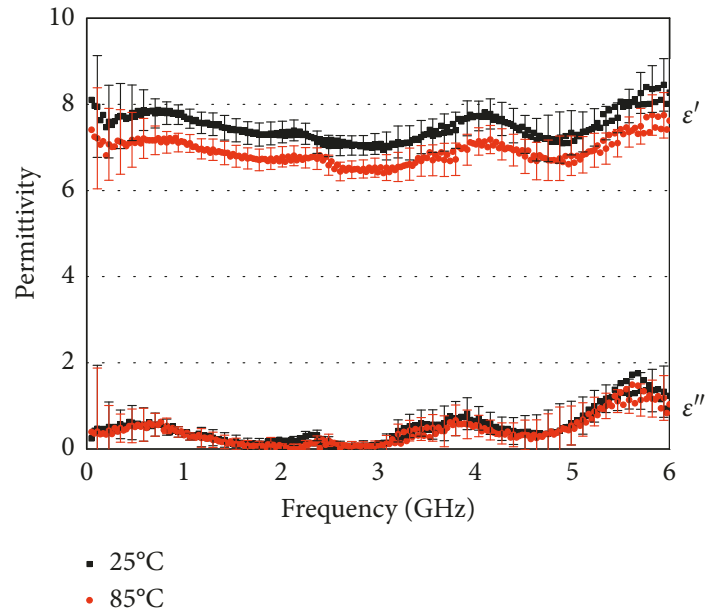

(a)

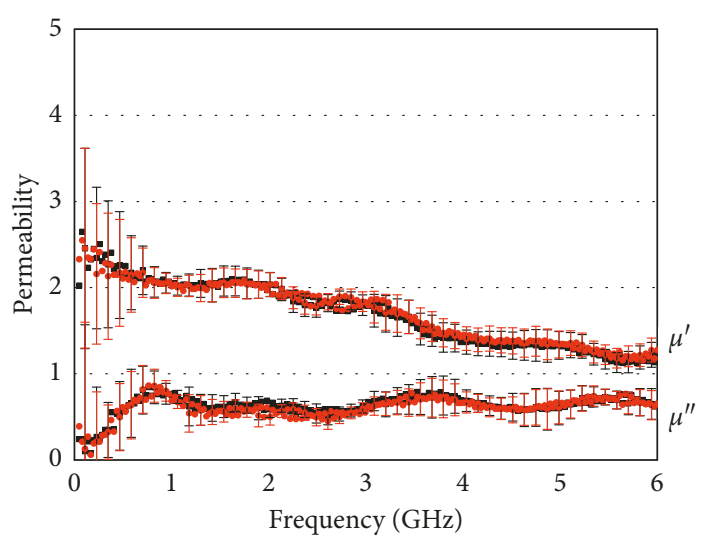

- $25^{\circ} \mathrm{C}$

- $85^{\circ} \mathrm{C}$

(b)

FiguRe 6: Real part and imaginary part of permittivity (a) and permeability (b) of a sample of $4 \mathrm{~mm}$ length of APU10 with frequency and temperature variations with the corresponding error. The frequency range is from $50 \mathrm{MHz}$ to $6 \mathrm{GHz}$ at two different temperatures, $25^{\circ} \mathrm{C}$ and $85^{\circ} \mathrm{C}$.

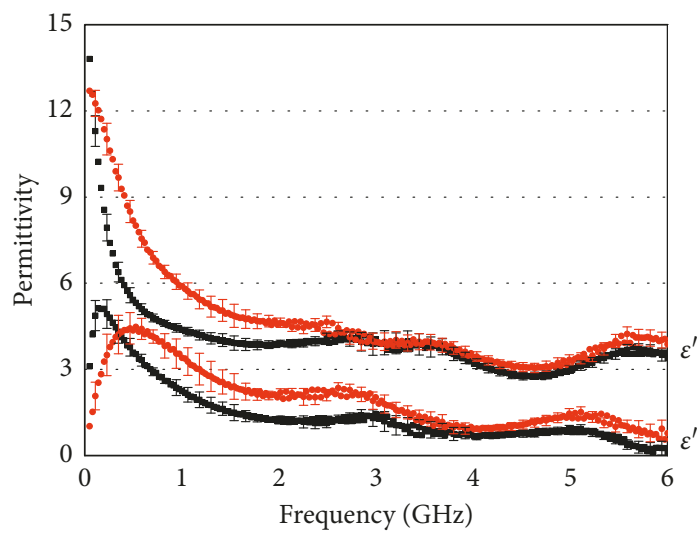

- $25^{\circ} \mathrm{C}$

- $45^{\circ} \mathrm{C}$

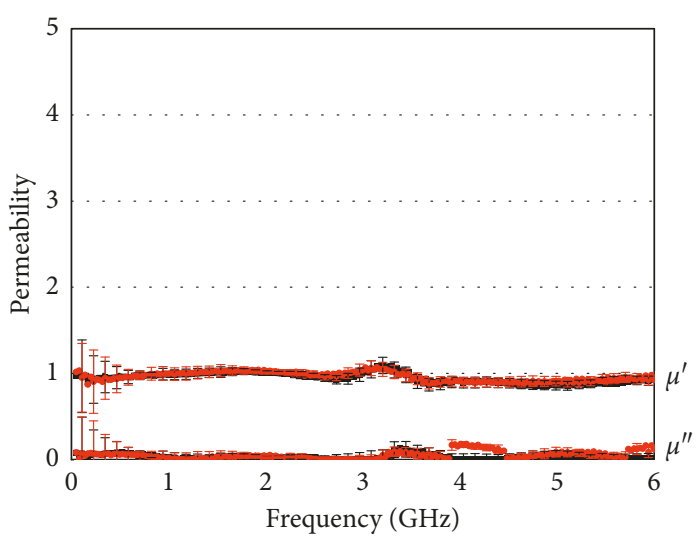

- $25^{\circ} \mathrm{C}$

- $45^{\circ} \mathrm{C}$

(a)

(b)

FIGURE 7: Real part and imaginary part of permittivity (a) and permeability (b) of a sample of cyclohexanol with frequency and temperature variations with the corresponding error. The frequency range is from $50 \mathrm{MHz}$ to $6 \mathrm{GHz}$ at two different temperatures, $25^{\circ} \mathrm{C}$ and $45^{\circ} \mathrm{C}$.

Two main effects of temperature measurement have been presented. Firstly, it is the cell dilation effect which may appear at high temperatures. In the actual configuration of the setup, the dilation effect was negligible throughout the temperature range. Secondly, it is the effect of sample temperature homogeneity, which is decisive when measuring the permittivity of samples of a certain considerable volume.

Finally, permittivity and permeability measurements have been conducted on different materials; as a solid material, APU10, which is magnetodielectric, has been chosen. The temperature variation behaviour of the material has been presented, as well as the frequency variation. Likewise, as a liquid material, a sample of cyclohexanol has been studied. This one was a dielectric material with a wellknown frequency dependence.

As shown in this paper, the setup is a helpful technique to obtain an approximation of the permittivity and the permeability of any kind of material. Furthermore, on a temperature range of $25^{\circ} \mathrm{C}$ to $85^{\circ} \mathrm{C}$, it is also possible to measure permittivity and permeability variations. The next challenge will be the proposition of an upgrade of this setup to reach higher temperatures with coaxial line to maintain the wide band frequency measurement.

\section{Data Availability}

The data used to support the findings of this study are available from the corresponding author upon request.

\section{Conflicts of Interest}

The authors declare that there are no conflicts of interest regarding the publication of this paper. 


\section{Acknowledgments}

This work was co-financed by DGA and "Club des partenaires (Aix-Marseille University)". This project has received funding from the European Union's Horizon 2020 research and innovation program under grant agreement No 736937.

\section{References}

[1] J. Krupka, "Frequency domain complex permittivity measurements at microwave frequencies," Measurement Science and Technology, vol. 17, no. 6, pp. R55-R70, 2006.

[2] É. Georget, R. Abdeddaim, and P. Sabouroux, "A quasiuniversal method to measure the electromagnetic characteristics of usual materials in the microwave range," Comptes Rendus Physique, vol. 15, no. 5, pp. 448-457, 2014.

[3] J. Krupka, "Split post dielectric resonators for measurements of the complex permittivity of laminar dielectric materials at microwave frequencies," in Proceedings of the Conference Workshop on Applied Radio Science (WARS'2002), Leura, Blue Mountains, NSW, Australia, February 2002.

[4] J. Krupka, K. Derzakowski, B. Riddle, and J. Baker-Jarvis, “A dielectric resonator for measurements of complex permittivity of low loss dielectric materials as a function of temperature," Measurement Science and Technology, vol. 9, no. 10, pp. 1751-1756, 1998.

[5] D. K. Ghodgaonkar, V. V. Varadan, and V. K. Varadan, “A free-space method for measurement of dielectric constants and loss tangents at microwave frequencies," IEEE Transactions on Instrumentation and Measurement, vol. 38, no. 3, pp. 789-793, 1989.

[6] P. Sabouroux, "Universal sample holder for measuring the electromagnetic properties of a dielectric and/or magnetic material," Patent WO/2012/164229, 2012.

[7] Y. Zhang, E. Li, J. Zhang, C. Yu, H. Zheng, and G. Guo, “A broadband variable-temperature test system for complex permittivity measurements of solid and powder materials," Review of Scientific Instruments, vol. 89, no. 2, article 024701, 2018.

[8] J. M. Catal-Civera, A. J. Cans, P. Plaza-Gonzlez, J. D. Gutirrez, B. Garca-Baos, and F. L. Pearanda-Foix, "Dynamic measurement of dielectric properties of materials at high temperature during microwave heating in a dual mode cylindrical cavity," IEEE Transactions on Microwave Theory and Techniques, vol. 63, no. 9, pp. 2905-2914, 2015.

[9] V. V. Varadan, R. D. Hollinger, D. K. Ghodgaonkar, and V. K. Varadan, "Free-space, broadband measurements of high-temperature, complex dielectric properties at microwave frequencies," IEEE Transactions on Instrumentation and Measurement, vol. 40, no. 5, pp. 842-846, 1991.

[10] A. P. Gregory, R. N. Clarke, and M. G. Cox, "Traceable measurement of dielectric reference liquids over the temperature interval $10-50^{\circ} \mathrm{C}$ using coaxial-line methods," Measurement Science and Technology, vol. 20, no. 7, article 075106, 2009.

[11] F. Buckley and A. A. Maryott, "Tables of dielectric dispersion data for pure liquids and dilute solutions," in National Bureau of Standards circular 564, National Bureau of Standards, Washington, DC, USA, 1958.

[12] J. Baker-Jarvis, E. J. Vanzura, and W. A. Kissick, "Improved technique for determining complex permittivity with the transmission/reflection method," IEEE Transactions on Microwave Theory and Techniques, vol. 38, no. 8, pp. 1096-1103, 1990.
[13] L. G. Hector and H. L. Schultz, "The dielectric constant of air at radiofrequencies," Physics, vol. 7, no. 4, pp. 133-136, 1936.

[14] B. Edln, "The refractive index of air," Metrologia, vol. 2, no. 2, pp. 71-80, 1966.

[15] Y. Wang, M. N. Afsat, and R. Grignon, "Complex permittivity and permeability of carbonyl iron powders at microwave frequencies," IEEE Antennas and Propagation Society, AP-S International Symposium (Digest), vol. 4, no. 1, 2003.

[16] K. S. Cole and R. H. Cole, "Dispersion and absorption in dielectrics i. alternating current characteristics," Journal of Chemical Physics, vol. 9, no. 4, pp. 341-351, 1941.

[17] M. Mohamad, Ahmad, and K. Yamada, "Superionic PbSnF4: a giant dielectric constant material," Applied Physics Letters, vol. 91, no. 5, article 052912, 2007.

[18] S. Sudo, N. Shinyashiki, Y. Kitsuki, and Y. Shin, "Dielectric relaxation time and relaxation time distribution of alcoholwater mixtures," Journal of Physical Chemistry A, vol. 106, no. 3, pp. 458-464, 2002.

[19] M. Y. Onimisi and T. Ikyumbur, "Comparative analysis of dielectric constant and loss factor of pure butan-1-ol and ethanol," American Journal of Condensed Matter physics, vol. 5, no. 3, pp. 69-75, 2015.

[20] S. Arrhenius, "On the reaction velocity of the inversion of cane sugar by acids," Zeitschrift fr Physikalische Chemie, vol. 4, pp. 226-248, 1889. 


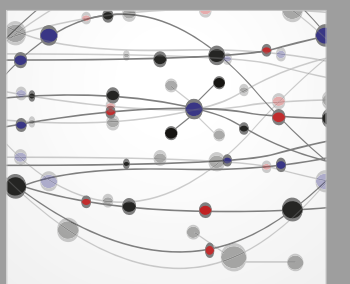

The Scientific World Journal
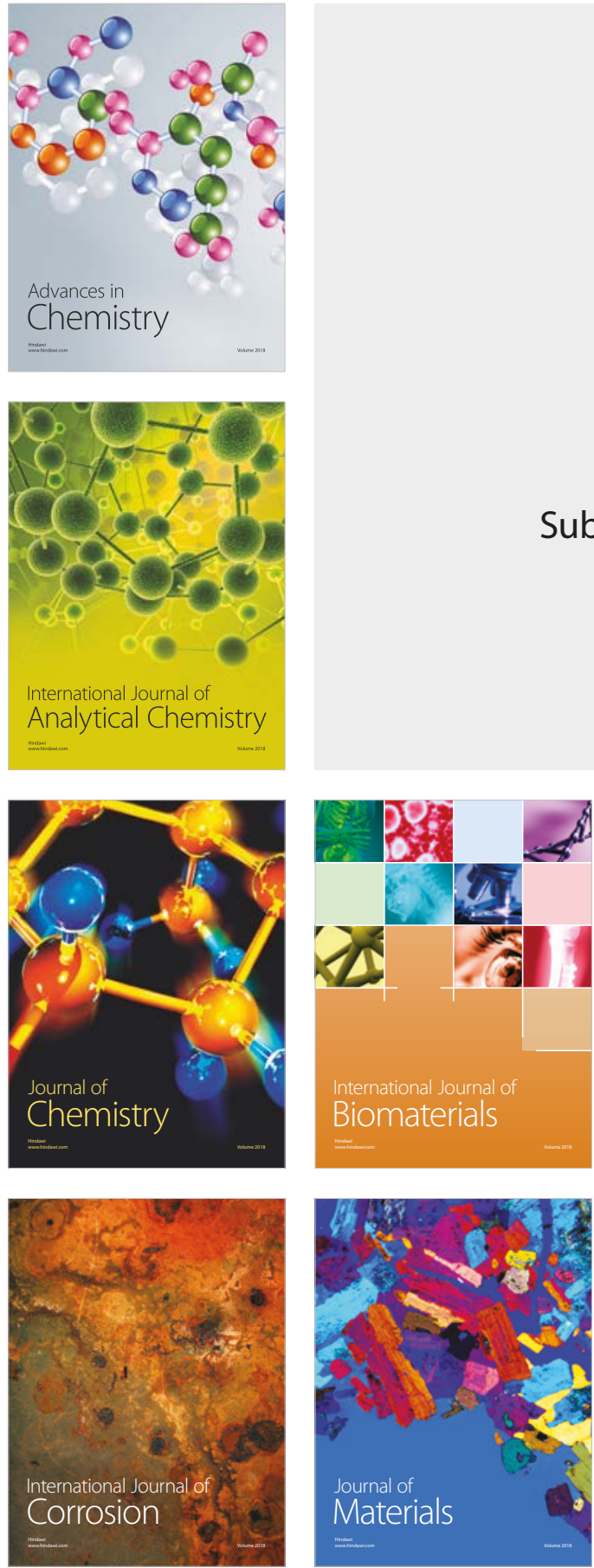

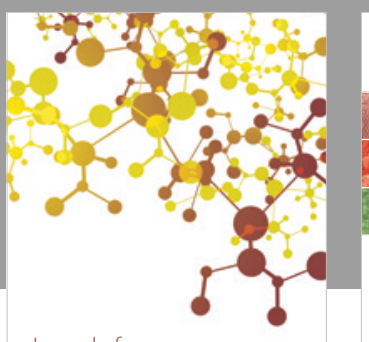

Journal of

Applied Chemistry
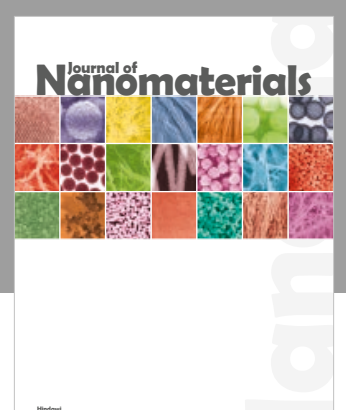

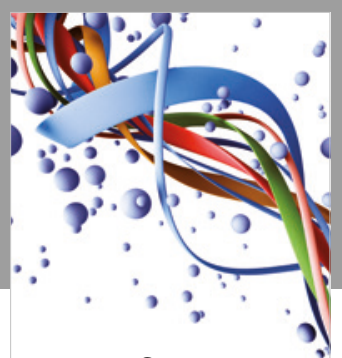

Scientifica

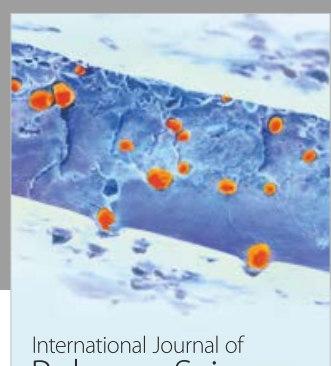

Polymer Science

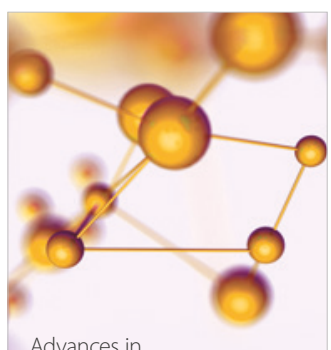

Physical Chemistry
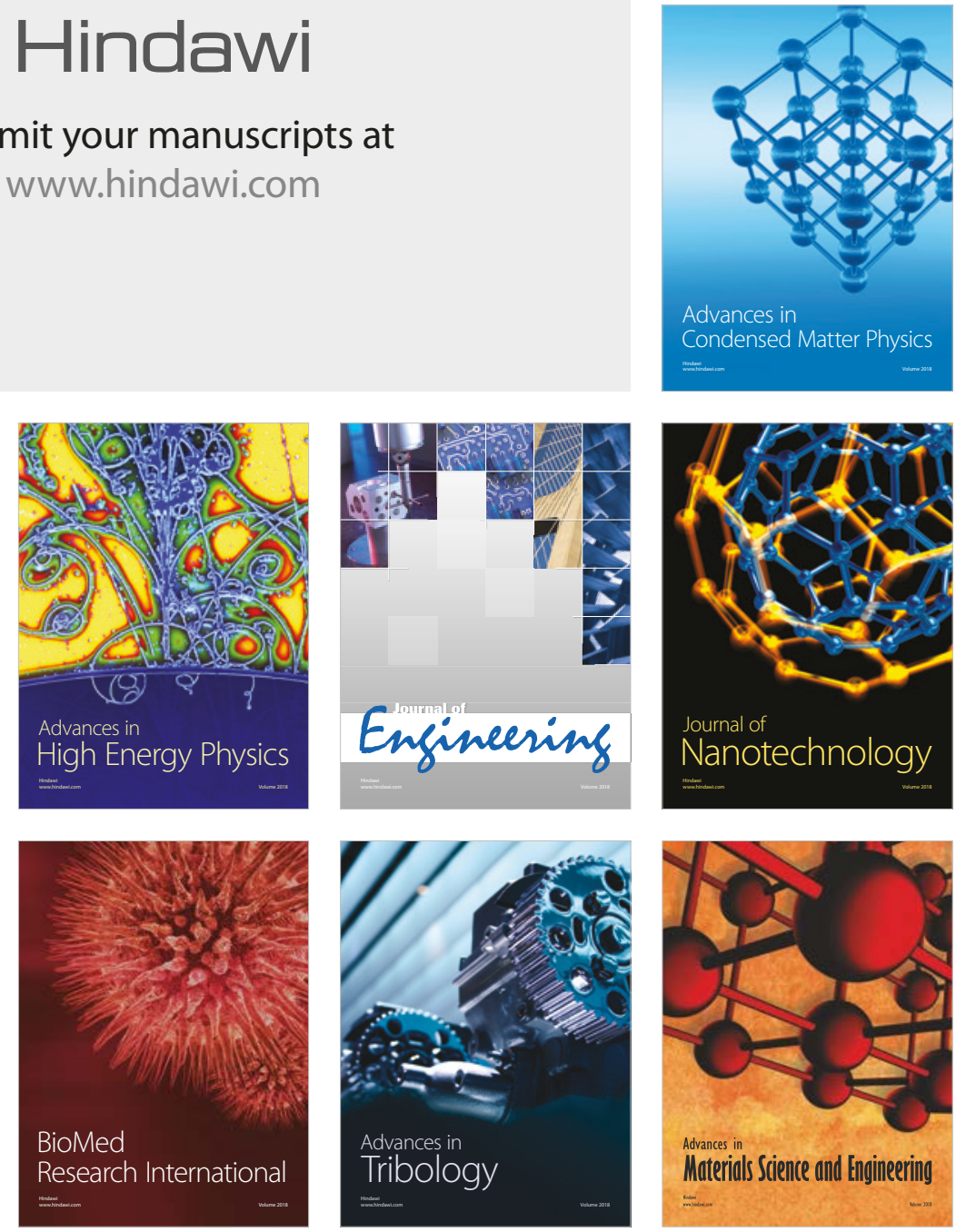